\title{
OSEEN FLOW OF A COMPRESSIBLE FLUID PAST A FLAT PLATE*
}

BY

\author{
DOREL HOMENTCOVSCHI
}

Central Institute of Mathematics, Bucharest

\begin{abstract}
The steady two-dimensional flow of a compressible fluid past a flat plate inclined to the uniform stream is studied on the basis of the Oseen approximation. The use of the momentum equations written in distributions allows us to obtain in a straightforward fashion the integral equations of the skin friction and of the lift. These equations are integrated for various values of the Reynolds and Mach numbers. The values obtained for the lift in the limit case of the inviscid fluid coincide with those known in conventional aerodynamics for the subsonic and for supersonic flow.
\end{abstract}

1. Introduction. The flow of the real fluid past a flat plate is one of the fundamental problems of fluid mechanics. The governing equations are the well-known Navier-Stokes equations. Since exact solutions for these equations were obtained only for very particular problems researchers resort to approximations. When the Reynolds number of the flow is very small the convective terms in Navier-Stokes equations are neglected with respect to the diffusive ones. The equations obtained, known as the Stokes approximation, work very well in three-dimensional problems of incompressible flow; but for two-dimensional flows this approximation fails to produce solutions. To avoid the "Stokes' paradox" Oseen replaced the convection terms in Navier-Stokes equations by convection due to free stream velocity. This is a good approximation at large distances, where the velocity perturbations are small, but can be seriously erroneous near the body. At low Reynolds numbers it is justified due to the dominance of the diffusion terms over the convective terms but at moderate and high Reynolds numbers the error may be significant, and the results obtained using the Oseen approximation are suspicious.

In the case of the motion past a flat plate with a small incidence angle $\theta$, it is possible to consider a slight modification of the Oseen approximation by taking the $x$-axis on the direction of the plate and linearizing the convection terms in Navier-Stokes equations, neglecting the product of perturbations and the product of perturbations by $\sin \theta$. The equations, similar in form to Oseen equations, are valid at large distances where the flow is almost uniform and coincide with the exact equations on the plate (due to no-slip conditions) for all values of the Reynolds number.

The two forms of the Oseen approximation produce the same value for the skin friction on the plate, but this value is unfortunately different from the correct one. Thus for the semi-infinite flat plate Carrier and Levis [6] pointed out the difference between the skin

* Received April 24, 1980; revised version received October 20, 1980. 
friction obtained by using Oseen's approximation and the Blasius one. To overcome this difficulty Carrier and Levis [6] and Sirovich [10] proposed some modifications which in fact consist in the use of Oseen equations too, but with different conditions at infinity and on the plate.

Concerning the lift, things are somewhat different, as we shall point out; the lift is different in the two Oseen approximations. The lift values obtained in the limiting case of the inviscid fluid by using the modified Oseen approximation coincide with those known in aerodynamics for the subsonic case as for the supersonic one. Moreover, in the subsonic case the analysis yields a mathematical justification for the Kutta-Joukowski condition.

In this paper the flow of a compressible viscous fluid past a flat plate is discussed on the basis of the modified Oseen approximation. Our study is based on the Navier-Stokes equations in distributions. The use of the mathematical tool of the theory of distributions allows us to obtain a representation for the velocity field in terms of the shear force and of the lift on the plate. Therefore the integral equations of the problem can be written directly. These equations are analyzed for various values of Reynolds and Mach numbers; for small values of the Reynolds number and $M_{0}^{2} \approx 2$ the solution is obtained by means of power series; for high values of these parameters the integral equations are solved asymptotically. From this point of view our analysis is an extension of the compressible flow in the results obtained by Bairstow, Cave and Lang [1], Piercy and Winny [8] and Miyagi [6] in studying incompressible motion past a flat plate.

We do not analyze the motion spectrum but, once the skin friction and the lift force on the plate are known, the velocity field can be obtained by using the asymptotic methods given in $[10]$.

2. Basic equations. In this paper we shall consider the form in distributions of the fluid mechanics equations. The discontinuous form of the Navier-Stokes equations was deduced by Sirovich $[10]$ :

$$
\begin{gathered}
\frac{\partial \rho}{\partial t}+\sum_{j=1}^{3} \frac{\partial}{\partial x_{j}}\left(\rho V_{j}\right)=\sum_{j=1}^{3}\left[\rho\left(V_{j}-d\right)\right] n_{j} \delta_{S} \\
\frac{\partial \rho V_{i}}{\partial t}+\sum_{j=1}^{3} \frac{\partial}{\partial x_{j}}\left(\rho V_{i} V_{j}\right)+\frac{\partial}{\partial x_{i}}(p-(\lambda+\mu) \operatorname{div} \mathbf{V}) \\
-\mu \Delta V_{i}=\left[T_{i}\right] \delta_{S}+\sum_{j=1}^{3}\left[\rho V_{i}\left(V_{j}-d\right)\right] n_{j} \delta_{S} .
\end{gathered}
$$

Here $S$ denotes a discontinuity surface of the fluid motion, $\mathbf{n}$ the exterior normal and $\mathbf{d}$ the displacement velocity of the surface $S$. Likewise the bracket operator represents the jump across $S$ and $\delta_{S}$ is the distribution

$$
\iiint \delta_{S} \varphi d \mathbf{x}=\iint_{S} \varphi d \sigma
$$

where $\varphi$ is a test function. The fluid motion is extended throughout the space by defining a source-free flow in the interior of the body; thus the body surface becomes a surface of discontinuity. If we assume no flow across the body surface, all terms but $\left[T_{i}\right] \delta_{s}$ on the right-hand side of relations (2.1), (2.2) vanish. The remaining term can be recognized as the action of the body on the two fluid motions. 
Eqs. (2.1), (2.2) are valid throughout the space; outside the discontinuity surface they coincide with the classical Navier-Stokes equations and for a fluid particle on the surface $S$ they describe the mass and momentum balance by taking into account the action of the body on the fluid flow.

In the inviscid limit Eqs. (2.1), (2.2) are also valid on a discontinuity surface without a material support inside the fluid. In this case all terms on the right-hand side of Eqs. (2.1), (2.2) vanish due to discontinuity relations resulting from integral form of the conservation laws. Consequently the motion equations in the discontinuity form contain only source terms corresponding to boundary surfaces [3].

The system of equations (2.1), (2.2) will be completed with the state equation of the gas. In isentropic flow this will be

$$
p / p_{0}=\left(\rho / \rho_{0}\right)^{\hat{\gamma}} .
$$

We consider a flat plate of infinite span and chord length $2 l$ immersed in a viscous compressible uniform flow of speed $\mathbf{V}_{0}$. The flat plate is assumed to be inclined at a small angle $\theta$ to the uniform stream. We take the rectangular coordinates $\left(x_{1}, x_{2}\right)$ in the plane of fluid motion in such a way that the $x_{1}$ axis is parallel to the direction of the plate and the origin coincides with the midpoint of the plate. The undisturbed flow is characterized by velocity $\mathbf{V}_{0}=V_{0} \cos \theta \mathbf{i}+V_{0} \sin \theta \mathbf{j}$, the pressure $p_{0}$ and the density $\rho_{0}$. By assuming small perturbations we shall linearize Eqs. (2.1)-(2.3) about the undisturbed state. Denoting by $\mathbf{V}^{\prime}$, $p^{\prime}, \rho^{\prime}$ the velocity, pressure and density perturbations respectively, we normalize our variables by using the following dimensionless quantities:

$$
x=\frac{x_{1}}{l}, \quad y=\frac{x_{2}}{l}, \quad \mathbf{v}=\frac{V^{\prime}}{V_{0}}, \quad \rho=\frac{\rho^{\prime}}{\rho_{0}}, \quad p=\frac{p^{\prime}}{\rho_{0} V_{0}^{2}} ; \quad \mathbf{t}=\frac{\mathbf{T}}{\rho_{0} V_{0}^{2}} .
$$

The governing equations become

$$
\begin{gathered}
\frac{\partial \rho}{\partial x}+\operatorname{div} \mathbf{v}=0 \\
\frac{\partial \mathbf{v}}{\partial x}+\operatorname{grad}\left(p-\frac{\sigma-\alpha}{2 \alpha \sigma} \operatorname{div} \mathbf{v}\right)-\frac{1}{2 \sigma} \Delta \mathbf{v}=\mathbf{t}(x) \delta(y) \\
\rho=M_{0}^{2} p
\end{gathered}
$$

where

$$
2 \sigma=\frac{l V_{0} \rho_{0}}{\mu}, \quad 2 \alpha=\frac{l V_{0} \rho_{0}}{\lambda+2 \mu}, \quad M_{0}=\frac{V_{0}}{a_{0}},
$$

$4 \sigma$ being therefore the Reynolds number, $a_{0}$ the sound velocity in the uniform flow and $M_{0}$ the Mach number. In the equation (2.6) the unknown density $t(x)$ vanishes outside the plate $(|x|>1)$.

The system of equations above must be completed with the conditions at infinity and boundary conditions. All perturbations must vanish at infinity:

$$
\lim _{x^{2}+y^{2} \rightarrow \infty}(\mathbf{v}, \rho, p)=0,
$$

and on the plate we consider the no-slip condition

$$
\begin{aligned}
& v_{x}(x, 0)=-1, \quad-1<x<1, \\
& v_{y}(x, 0)=-\theta, \quad-1<x<1 .
\end{aligned}
$$


The Oseen linearization used in this paper is different from that used by Miyagi [7]. In Miyagi's paper the $\mathrm{Ox}_{1}$-axis is taken in the direction of the undisturbed velocity $\mathbf{V}_{0}$; the linearized equations are identical with Eqs. (2.5), (2.6) but the boundary conditions are different. We prefer to consider the motion "with respect to the plate" since in this case the Navier-Stokes equations on the plate are identical with the linearized ones.

3. The integral equations of the problem. Denoting the Fourier transforms by capital letters

$$
\begin{aligned}
& \mathbf{V}\left(k_{1}, k_{2}\right)=\mathscr{F}[\mathbf{v}(x, y)] \equiv \iiint_{-\infty}^{\infty} \mathbf{v}(x, y) e^{-i\left(k_{1} x+k_{2} y\right)} d x d y, \\
& P\left(k_{1}, k_{2}\right)=\mathscr{F}[p(x, y)], \quad \mathbf{T}\left(k_{1}\right)=\mathscr{F}[\mathbf{t}(x) \delta(y)],
\end{aligned}
$$

and applying the Fourier transform with respect to $x$ and $y$ to the system $(2.5)-(2.7)$, we obtain

$$
\begin{gathered}
i k_{1} P+M_{0}^{-2} i \mathbf{k} \cdot \mathbf{V}=0 \\
i k_{1} \mathbf{V}+i \mathbf{k}\left(P+\frac{\alpha-\sigma}{2 \alpha \sigma}(i \mathbf{k} \cdot \mathbf{V})\right)+\frac{1}{2 \sigma} k^{2} \mathbf{V}=\mathbf{T}\left(k_{1}\right) \\
\left(k^{2}=|\mathbf{k}|^{2}=k_{1}^{2}+k_{2}^{2}\right) .
\end{gathered}
$$

The solution of this system is

$$
\begin{aligned}
P\left(k_{1}, k_{2}\right) & =\frac{i \mathbf{k} \cdot \mathbf{T}\left(k_{1}\right)}{M_{0}^{2} k_{1}^{2}-k^{2}-\frac{i k_{1} k^{2}}{2 \alpha} M_{0}^{2}}, \\
V_{x}\left(k_{1}, k_{2}\right) & =K_{11}\left(k_{1}, k_{2}\right) \cdot T_{1}\left(k_{1}\right)+K_{12}\left(k_{1}, k_{2}\right) \cdot T_{2}\left(k_{1}\right), \\
V_{y}\left(k_{1}, k_{2}\right) & =K_{21}\left(k_{1}, k_{2}\right) \cdot T_{1}\left(k_{1}\right)+K_{22}\left(k_{1}, k_{2}\right) \cdot T_{2}\left(k_{1}\right),
\end{aligned}
$$

where

$$
\begin{aligned}
K_{11}\left(k_{1}, k_{2}\right)= & \frac{2 \sigma-i k_{1}}{k^{2}+2 \sigma i k_{1}}+\frac{i k_{1}}{k^{2}-\frac{2 \alpha k_{1}^{2} M_{0}^{2}}{2 \alpha+i k_{1} M_{0}^{2}}}, \\
K_{12}\left(k_{1}, k_{2}\right)= & K_{21}\left(k_{1}, k_{2}\right)=\frac{-i k_{2}}{k^{2}+2 \sigma i k_{1}}+\frac{i k_{2}}{k^{2}-\frac{2 \alpha k_{1}^{2} M_{0}^{2}}{2 \alpha+i k_{1} M_{0}^{2}}}, \\
K_{22}\left(k_{1}, k_{2}\right)= & \frac{i k_{1}}{k^{2}+2 \sigma i k_{1}}-\frac{i k_{1}}{k^{2}-\frac{2 \alpha k_{1}^{2} M_{0}^{2}}{2 \alpha+i k_{1} M_{0}^{2}}} \\
& +\frac{2 \alpha i k_{1} M_{0}^{2}}{\left(2 \alpha+i k_{1} M_{0}^{2}\right)\left(k^{2}-\frac{2 \alpha k_{1}^{2} M_{0}^{2}}{2 \alpha+i k_{1} M_{0}^{2}}\right)}
\end{aligned}
$$


By taking the inverse Fourier transform of the relations (3.6), (3.7) we obtain the following representation for the velocity field:

$$
\begin{aligned}
& v_{x}(x, y)=k_{11}(x, y) * t_{1}(x)+k_{12}(x, y) * t_{2}(x), \\
& v_{y}(x, y)=k_{21}(x, y) * t_{1}(x)+k_{22}(x, y) * t_{2}(x),
\end{aligned}
$$

where $*$ denotes the convolution product with respect to the variable $x$. In relations (3.11), (3.12) we have put

$$
\begin{aligned}
k_{11}(x, y) & =\frac{\sigma}{2 \pi} e^{\sigma x}\left(K_{0}(\sigma r)+\frac{x}{r} K_{1}(\sigma r)\right)+\frac{i}{4 \pi} \int_{-\infty}^{+\infty} \frac{\operatorname{sgn} k_{1}}{R} e^{i k_{1} x-\left|y k_{1}\right| R} d k_{1}, \\
k_{12}(x, y) & =\frac{\sigma}{2 \pi} e^{\sigma x} \frac{y}{r} K_{1}(\sigma r)-\frac{\operatorname{sgn} y}{4 \pi} \int_{-\infty}^{\infty} e^{i k_{1} x-\left|y k_{1}\right| R} d k_{1}=k_{21}(x, y), \\
k_{22}(x, y) & =\frac{\sigma}{2 \pi} e^{\sigma x}\left(K_{0}(\sigma r)-\frac{x}{r} K_{1}(\sigma r)\right)-\frac{i}{4 \pi} \int_{-\infty}^{\infty} \frac{\operatorname{sgn} k_{1}}{R} e^{i k_{1} x-\left|y k_{1}\right| R} d k_{1} \\
& +\frac{\alpha i}{\pi} \int_{-\infty}^{+\infty} \frac{M_{0}^{2} \operatorname{sgn} k_{1}}{\left(2 \alpha+i k_{1} M_{0}^{2}\right) R} e^{i k_{1} x-\left|y k_{1}\right| R} d k_{1} .
\end{aligned}
$$

Here

$$
r=\left(x^{2}+y^{2}\right)^{1 / 2}, \quad R=\left(\frac{2 \alpha\left(1-M_{0}^{2}\right)+i k_{1} M_{0}^{2}}{2 \alpha+i k_{1} M_{0}^{2}}\right)^{1 / 2} .
$$

The square root determination in $R$ is the one with positive real part for real $k_{1}$.

In order to satisfy the boundary relations $(2.13),(2.14)$ we must estimate the functions $k_{11}(x, 0), k_{12}(x, 0)$ and $k_{22}(x, 0)$. The Fourier integrals of the form appearing in these functions were calculated by us previously [4]. We have

$$
\begin{aligned}
k_{11}(x) \equiv & k_{11}(x, 0)=\frac{\sigma}{2 \pi} e^{\sigma x}\left(K_{0}(\sigma|x|)+\operatorname{sgnx} K_{1}(\sigma|x|)\right) \\
& +\frac{\alpha}{2 \pi} e^{-\alpha \gamma x}\left\{K_{0}(\alpha|x|)-\operatorname{sgnx} K_{1}(\alpha|x|)\right. \\
& \left.-\left[K_{0}(-\alpha x, \gamma)+K_{1}(-\alpha x, \gamma)\right] H\left(1-M_{0}\right)\right\}, \\
k_{12}(x, 0)= & k_{21}(x, 0)=0, \\
k_{22}(x) \equiv & k_{22}(x, 0)=\frac{\sigma}{2 \pi} e^{\sigma x}\left(K_{0}(\sigma|x|)-\operatorname{sgnx} K_{1}(\sigma|x|)\right) \\
& +\frac{\alpha}{2 \pi} e^{-\alpha \gamma x}\left\{K_{0}(\alpha|x|)+\operatorname{sgnx} K_{1}(\alpha|x|)\right. \\
& \left.-\left[K_{0}(-\alpha x, \gamma)-K_{1}(-\alpha x, \gamma)\right] H\left(1-M_{0}\right)\right\},
\end{aligned}
$$

where $H(x)$ is the Heaviside function and $\gamma=2 M_{0}^{-2}-1$. We also denote

$$
K_{j}(x, \gamma)=\int_{1}^{\gamma} \frac{t^{j} e^{-x t}}{\left[t^{2}-1\right]^{1 / 2}} d t
$$


for the incomplete cylindrical functions of the second kind. $K_{j}(x)=K_{j}(x, \infty)$ are Macdonald functions.

The boundary conditions now give

$$
\begin{aligned}
& \int_{-1}^{1} k_{11}(x-\xi) t_{1}(\xi) d \xi=-1, \quad-1<x<1, \\
& \int_{-1}^{1} k_{22}(x-\xi) t_{2}(\xi) d \xi=-\theta, \quad-1<x<1 .
\end{aligned}
$$

Thus, for determining the mechanical parameters of interest (the shear force $t_{1}(x)$ and the lift $t_{2}(x)$ ), we obtain two decoupled regular integral equations of the first kind. The form of these equations is similar to the integral equation obtained by Bairstow, Cave and Lang [1] for the case of the incompressible fluids. Consequently the methods used to solve the integral equations (3.19), (3.20) will be similar to those used to obtain the solution of Bairstow's equation.

4. Series solutions of the integral equations for small and moderate values of $\sigma$ and $\gamma$. To obtain results valid for small and moderate values of the parameters $\sigma$ and $\gamma$ we follow the procedure suggested by Piercy and Winny [8] for the incompressible case. We assume that we may write

$$
\begin{aligned}
& t_{1}(x)=\left(1-x^{2}\right)^{-1 / 2} \sum_{s=0}^{\infty} A_{s}^{(1)} x^{s}, \\
& t_{2}(x)=\left(1-x^{2}\right)^{-1 / 2} \theta \sum_{s=0}^{\infty} A_{s}^{(2)} x^{s},
\end{aligned}
$$

where $A_{s}^{(1)}, A_{s}^{(2)}$ are constants which must be determined. We expand the kernels $k_{11}(x)$ and $k_{22}(x)$ in series of $x^{n}$ and $x^{n} \log |x|$ :

$$
\begin{aligned}
& k_{11}(x)=\frac{1}{\pi} \sum_{j=0}^{\infty}\left(Z_{1 j} x^{j} \log 2|x|+Z_{2 j} x^{j}\right), \\
& k_{22}(x)=\frac{1}{\pi} \sum_{j=0}^{\infty}\left(Z_{3 j} x^{j} \log 2|x|+Z_{4 j} x^{j}\right),
\end{aligned}
$$

where

$$
\begin{aligned}
& Z_{1 j}=\frac{\sigma}{2} \sum_{r=0}^{j} \frac{\sigma^{j-r} a_{r}(\sigma)}{(j-r) !}+(-1)^{j} \frac{\alpha}{2} \sum_{r=0}^{j} \frac{a_{r}(\alpha)(\alpha \gamma)^{j-r}}{(j-r) !} \\
Z_{2 j}= & \frac{\sigma}{2}\left\{\frac{\sigma^{j}}{(j+1) !}+\sum_{r=0}^{j} \frac{\sigma^{j-r} b_{r}(\sigma)}{(j-r) !}\right\}+(-1)^{j} \frac{\alpha}{2} \\
& \cdot\left\{\frac{\sigma^{j} \gamma^{j+1}}{(j+1) !}+\sum_{r=0}^{j} \frac{(\alpha \gamma)^{j-r}}{(j-r) !} \cdot\left[b_{r}(\alpha)-\frac{(-1)^{r} \alpha^{r}}{r !}\left(Y_{r}+Y_{r+1}\right) H\left(1-M_{0}\right)\right]\right\}, \\
& Z_{3 j}=\frac{\sigma}{2} \sum_{r=0}^{j}(-1)^{r} \frac{\sigma^{j-r} a_{r}(\sigma)}{(j-r) !}+(-1)^{j} \frac{\alpha}{2} \sum_{r=0}^{j} \frac{(-1)^{r}(\alpha \gamma)^{j-r} a_{r}(\alpha)}{(j-r) !},
\end{aligned}
$$




$$
\begin{aligned}
Z_{4 j}= & \frac{\sigma}{2}\left\{-\frac{\sigma^{j}}{(j+1) !}+\sum_{r=0}^{j}(-1)^{r} \frac{\sigma^{j-r} b_{r}(\sigma)}{(j-r) !}\right\}+(-1)^{j} \frac{\alpha}{2}\left\{-\frac{\alpha^{j} \gamma^{j+1}}{(j+1) !}+\sum_{r=0}^{j} \frac{(-1)^{r}(\alpha \gamma)^{j-r}}{(j-r) !}\right. \\
& \left.\cdot\left[b_{r}(\alpha)+\frac{\alpha^{r}}{r !}\left(Y_{r+1}-Y_{r}\right) H\left(1-M_{0}\right)\right]\right\} .
\end{aligned}
$$

The functions $a_{r}(\sigma), b_{r}(\sigma)$ coincide with those given in [9]:

$$
\begin{aligned}
a_{2 n}(\sigma) & =-\frac{\sigma^{2 n}}{2^{2 n}(n !)^{2}}, \\
a_{2 n+1}(\sigma) & =\frac{\sigma^{2 n+1}}{2^{2 n+1} n !(n+1) !}, \\
b_{2 n}(\sigma) & =a_{2 n}(\sigma)[\log (0.25 \sigma)-\psi(n+1)], \\
b_{2 n+1}(\sigma) & =a_{2 n+1}(\sigma)\left[\log (0.25 \sigma)-\psi(n+1)-\frac{1}{2(n+1)}\right],
\end{aligned}
$$

where $\psi(n)$ is the psi function. Likewise the coefficients $Y_{r}$ are given by recurrence relations:

$$
\begin{aligned}
& Y_{0}=\log \left(\gamma+\sqrt{\gamma^{2}-1}\right), \quad Y_{1}=\sqrt{\gamma^{2}-1}, \\
& Y_{j}=\frac{j-1}{j} Y_{j-2}+\frac{\gamma^{j-1}}{j} \sqrt{\gamma^{2}-1} \quad(j \geq 2) .
\end{aligned}
$$

The series (4.1) $-(4.4)$ are substituted into the integral equations (3.19) $-(3.20)$ and the integration is performed term by term. To estimate the integrals which arise in this computation we use the formulae given in [11]:

$$
\begin{array}{ll}
\frac{1}{\pi} \int_{-1}^{1} \frac{\xi^{n}}{\sqrt{1-\xi^{2}}} \frac{d \xi}{\xi-x} & =\sum_{r=0}^{n-1} I_{n-r-1} x^{r} \quad(n=0,1,2, \ldots), \\
\frac{1}{\pi} \int_{-1}^{1} \frac{\xi^{n}(x-\xi)^{m}}{\sqrt{1-\xi^{2}}} d \xi & =\sum_{r=0}^{m} b_{r}(m, n) x^{r} \quad(m, n=0,1,2, \ldots),
\end{array}
$$

Here

$$
\begin{gathered}
b_{r}(m, n)=(-1)^{m-r}\left(\begin{array}{c}
m \\
r
\end{array}\right) I_{n+m-r}, \\
\frac{1}{\pi} \int_{-1}^{1} \frac{\xi^{n}(x-\xi)^{m} \log 2|x-\xi|}{\sqrt{1-\xi^{2}}} d \xi=\sum_{r=0}^{m} C_{r}(m, n) x^{r} \quad(m, n=0,1,2, \ldots)
\end{gathered}
$$

where

$$
\begin{aligned}
C_{r}(m, n) & =(-1)^{m-r}\left(\begin{array}{c}
m \\
r
\end{array}\right) I_{n+m-r}\left\{\sum_{t=1}^{n+m-r} \frac{(-1)^{t}}{t}+\sum_{t=0}^{r-1} \frac{1}{m-t}, \quad 0 \leq r \leq m\right. \\
& =-\frac{m !(r-m-1) !}{r !} I_{n+m-r}, \quad m+1 \leq r \leq m+n .
\end{aligned}
$$

Here $I_{0}=0, I_{2 n}=(2 n) ! /\left[2^{2 n}(n !)^{2}\right], n=1,2, \ldots, I_{2 n+1}=0$. 
When we insert these formulae into (3.19), (3.20) the integral equations yield two power series in $x$ which must be identical to -1 for any $x$ :

$$
\begin{aligned}
& \sum_{r=0}^{\infty} x^{r} \sum_{n=0}^{\infty} A_{n}^{(1)} \sum_{m=0}^{\infty}\left\{Z_{1 m} C_{r}(m, n)+Z_{2 m} b_{r}(m, n)\right\}=-1, \\
& \sum_{r=0}^{\infty} x^{r} \sum_{n=0}^{\infty} A_{n}^{(2)} \sum_{m=0}^{\infty}\left\{Z_{3 m} C_{r}(m, n)+Z_{4 m} b_{r}(m, n)\right\}=-1 .
\end{aligned}
$$

The undefined values of the coefficients are zero. By matching the two sides of the relations (4.12), (4.13) we obtain two sets of infinite linear equations in the unknowns $A_{n}^{(1)}, A_{n}^{(2)}$ $(n=0,1,2, \ldots)$.

The forces acting on the plate can be obtained by means of the coefficients $A_{n}^{(j)}$ :

$$
\begin{aligned}
& D=-\rho_{0} V_{0}^{2} l \int_{-1}^{1} t_{1}(x) d x=-\pi \rho_{0} V_{0}^{2} l \sum_{j=0}^{\infty} \frac{(2 j) !}{2^{2 j}(j !)^{2}} A_{2 j}^{(1)}, \\
& L=-\rho_{0} V_{0}^{2} l \int_{-1}^{1} t_{2}(x) d x=-\rho_{0} \pi V_{0}^{2} \theta l \sum_{j=0}^{\infty} \frac{(2 j) !}{2^{2 j}(j !)^{2}} A_{2 j}^{(2)} .
\end{aligned}
$$

For numerical computation the sums in (4.11), (4.12) are truncated at a value of $n$ and $m$, say $N$, such that the inner sums in these relations are convergent and the subsequent $A_{n}$ 's are negligible. We took $N=15$ and computed the solution in the Stokes approximation $3 \lambda+2 \mu=0$ for values of the Reynolds number $\operatorname{Re}=0.1-2$, and for values of the Mach number $M_{0}=0.8,1.33,2$. Numerical results for the drag coefficient $C_{D}$ and the lift coefficient $C_{L}$ written as

$$
\begin{aligned}
& C_{D}=\frac{D}{2 \rho_{0} V_{0}^{2} l}, \\
& C_{L}=\frac{L}{2 \rho_{0} V_{0}^{2} l} \cdot \frac{1}{\theta^{\text {(degree })}}
\end{aligned}
$$

are given in Figs. 1 and 2 respectively. By taking greater values of $N$ the computation can be extended to moderate values of the coefficients $\sigma$ and $\gamma$.

5. Solution for small Mach numbers. In the case of small Mach numbers, we consider the asymptotic expansions of the kernels of the equations (3.19), (3.20) for large values of the parameter $\gamma$. For $x<0$ we have

$$
\begin{gathered}
e^{-\alpha \gamma x}\left\{K_{j}(\alpha|x|)-K_{j}(-\alpha x, \gamma)\right\}=\gamma^{j} \int_{1}^{\infty} u^{j-1} e^{\alpha \gamma x(u-1)} d u+\frac{\gamma^{j-2}}{2} \int_{1}^{\infty} \frac{e^{\alpha \gamma x(u-1)}}{u^{3-j}} d u \\
\left.+\gamma^{j} \int_{1}^{\infty} u^{j} e^{\alpha \gamma x(u-1)} \mid \frac{1}{\mid \sqrt{u^{2}-\frac{1}{\gamma^{2}}}}-\frac{1}{u}-\frac{1}{2 \gamma^{2} u^{3}}\right\} d u .
\end{gathered}
$$


$C_{D}$

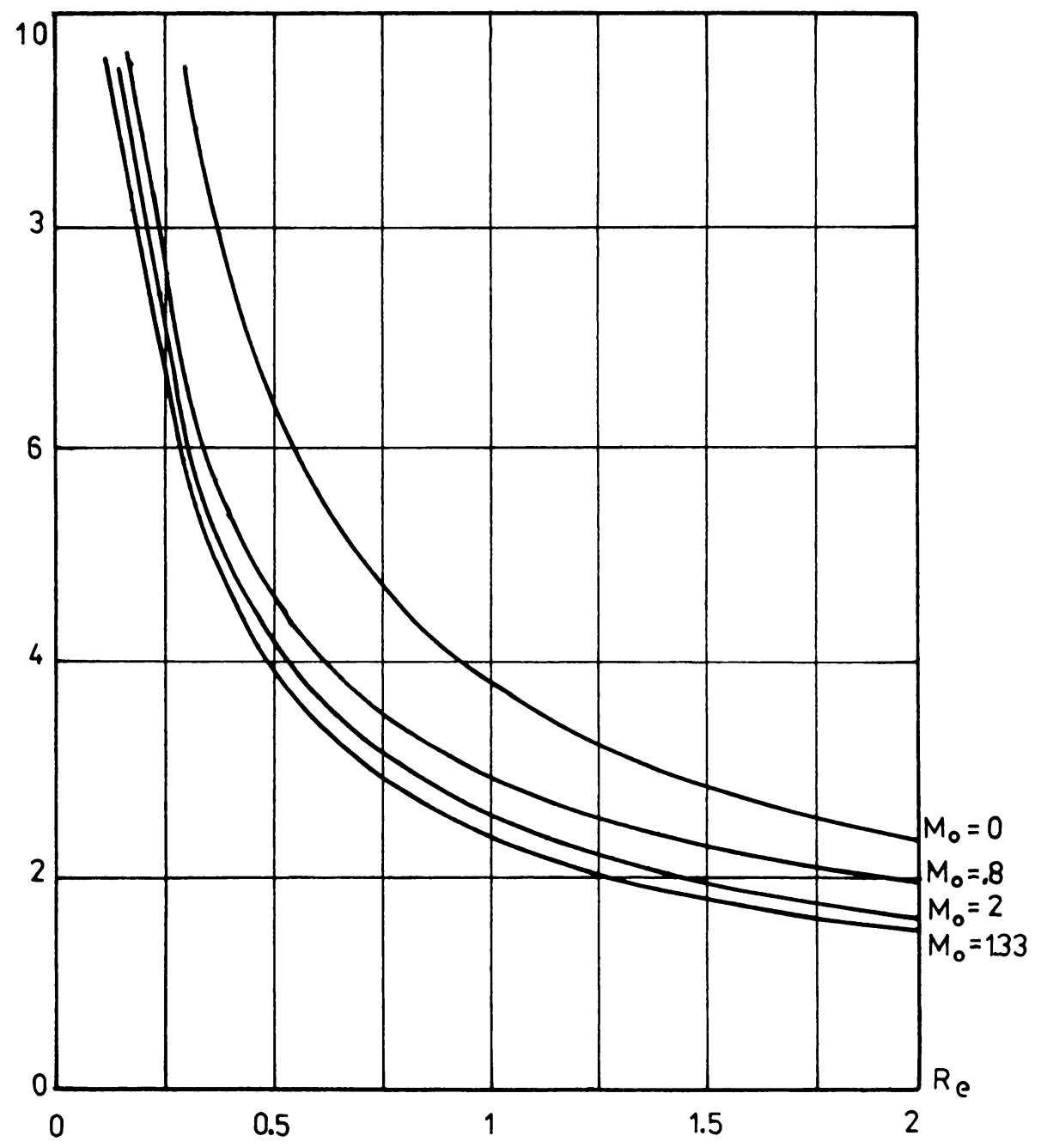

Fig. 1. Drag coefficient $C_{D}$ plotted against Reynolds number Re for various Mach numbers $\boldsymbol{M}_{0}$. 


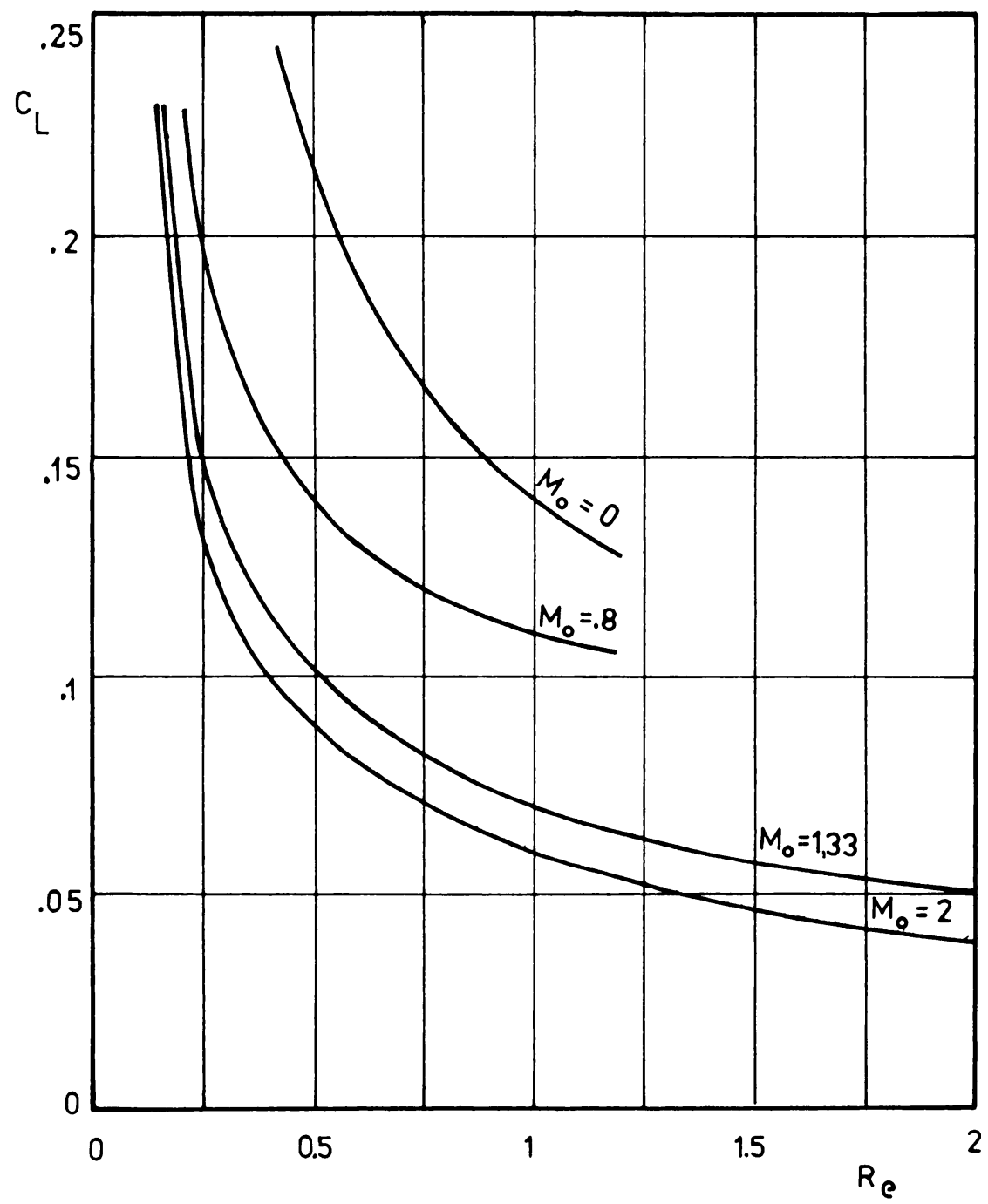

FIG. 2. Lift coefficient $C_{L}$ plotted against Reynolds number Re for various Mach numbers $M_{0}$. 
The last integral in (5.1) is $O\left(\gamma^{k-4}\right)$ and after several integrations by parts from (5.1) we obtain

$$
e^{-\alpha \gamma x}\left\{K_{j}(\alpha|x|)-K_{j}(-\alpha x, \gamma)\right\}=-\frac{1}{\alpha \gamma^{1-j} x}+O\left(\frac{1}{\gamma^{2}}\right) \quad(j=0,1) .
$$

For $x>0$ we can write

$$
\begin{aligned}
e^{-\alpha \gamma x} K_{j}(-\alpha x, \gamma)= & e^{-\alpha \gamma x}\left\{\gamma^{j} \int_{1 / 2}^{1} u^{j-1} e^{\alpha \gamma x u} d u+\frac{\gamma^{j-2}}{2} \int_{1 / 2}^{1} u^{j-3} e^{\alpha \gamma x u} d u\right. \\
& +\gamma^{j} \int_{1 / 2}^{1} u^{j} e^{\alpha \gamma x u}\left\{\frac{1}{\sqrt{u^{2}-\frac{1}{\gamma^{2}}}}-\frac{1}{u}-\frac{1}{2 \gamma^{2} u^{3}}\right\} d u \mid+O\left(\gamma e^{-\alpha \gamma x / 2}\right)
\end{aligned}
$$

and hence

$$
e^{-x \gamma x} K_{j}(-\alpha x, \gamma)=\frac{1}{\alpha \gamma^{1-j} x}+O\left(\frac{1}{\gamma^{2}}\right)
$$

Now, by using the obtained asymptotic expansions, we can write the kernels of the integral equations (3.19), (3.20) as

$$
\begin{aligned}
& k_{11}(x)=\frac{\sigma}{2 \pi} e^{\sigma x}\left(K_{0}(\sigma|x|)+\operatorname{sgn} x K_{1}(\sigma|x|)\right)-\frac{1}{2 \pi x}\left(1+\frac{1}{\gamma}\right)+O\left(\frac{1}{\gamma^{2}}\right), \\
& k_{22}(x)=\frac{\sigma}{2 \pi} e^{\sigma x}\left(K_{0}(\sigma|x|)-\operatorname{sgn} x K_{1}(\sigma|x|)\right)+\frac{1}{2 \pi x}\left(1-\frac{1}{\gamma}\right)+O\left(\frac{1}{\gamma^{2}}\right) .
\end{aligned}
$$

For zero Mach number $(\gamma \rightarrow \infty)$ the asymptotic forms of the integral equations of the problem are also regular integral equations. Eq. (3.19) reduces in this case to Bairstow's equation. We computed the coefficients $C_{D}$ and $C_{L}$ for $M_{0}=0$ by using the method of the previous section and have plotted the results also in Figs. 1 and 2.

In order to compare our results with those given by Miyagi [7], we calculated by hand the first approximation of the solution corresponding to the incompressible case. We have

$$
\begin{aligned}
C_{D} & =\frac{\pi}{\sigma\left(1-\ln \frac{\sigma}{4}-C\right)}, \\
C_{L} & =-\frac{\pi}{\sigma\left(1+\ln \frac{\sigma}{4}+C\right)} \cdot \frac{\pi}{180},
\end{aligned}
$$

where $C=0.577 \ldots$ is the Euler constant. The expression (5.6) for $C_{D}$ coincides with that given by Miyagi, but the lift coefficient is different. This is due to the different approximations performed in linearizing the Navier-Stokes equations, as discussed in the last part of Sec. 2.

In order to obtain asymptotic solutions of Eqs. (3.19), (3.20) for small Mach numbers the 
approximations (5.4), (5.5) of the kernels are to be used. The resulting integral equations are singular, with Cauchy finite-part singularities, but the method of series expansion can be still applied. The singular integrals will be estimated by means of the formula (4.9).

6. Asymptotic solutions for high Reynolds numbers in subsonic flow. To determine the asymptotic form of the functions $t_{1}(x)$ and $t_{2}(x)$ in the limit case of the inviscid fluid, we use the asymptotic expansions of the functions $K_{f}(z, \gamma), K_{f}(z)$ :

$$
\begin{aligned}
K_{j}(z, \gamma) & =e^{-z} \sqrt{\frac{\pi}{2 z}}-\frac{1}{z} \frac{\gamma^{j}}{\sqrt{\gamma^{2}-1}} e^{-\gamma z}+o\left(\frac{1}{z^{2}}\right), \\
K_{j}(z) & =e^{-z} \sqrt{\frac{\pi}{2 z}}\left\{1+\frac{4 j^{2}-1}{8 z}+O\left(\frac{1}{z^{2}}\right)\right\} \quad(j=0,1) .
\end{aligned}
$$

The asymptotic forms of the function $K_{f}(z)$ are given in the literature and to obtain the asymptotic form for the incomplete Bessel function $K_{j}(z, \gamma)$ we applied the standard asymptotic methods to the integral (3.18).

Now, the kernels of the integral equations (3.19), (3.20) become

$$
\begin{aligned}
& k_{11}(x)=\frac{\sqrt{ } \sigma}{\sqrt{2 \pi x}} H(x)-\frac{1}{2 \pi \beta x}+O\left(\frac{1}{\sqrt{ } \sigma}\right), \\
& k_{22}(x)=-\frac{H(x)}{4 x \sqrt{2 \pi \sigma x}}+\frac{\beta}{2 \pi x}+O\left(\frac{1}{\sigma}\right),
\end{aligned}
$$

where $\beta=\left(1-M_{0}^{2}\right)^{1 / 2}$.

i) The problem of drag. By using the simplified kernel (6.3), Eq. (4.19) becomes

$$
\sqrt{\frac{\sigma}{2 \pi}} \int_{-1}^{x} \frac{t_{1}(\xi)}{\sqrt{x-\xi}} d \xi-\frac{1}{2 \pi \beta} \int_{-1}^{1} \frac{t_{1}(\xi)}{x-\xi} d \xi=-1 .
$$

For $\beta=1$ Eq. (6.5) coincides with the equation given for the incompressible case. Its solution can be written in the form

$$
t_{1}(x)=-\frac{2}{\sqrt{2 \pi \sigma(x+1)}}-\frac{2 \sqrt{2}}{\beta \sigma \pi^{2} \sqrt{1-x^{2}}} \frac{\sin ^{-1} \sqrt{\frac{1+x}{2}}}{\sqrt{1+x}}+O\left(\frac{1}{\sigma \sqrt{ } \sigma}\right) .
$$

The ratio of the second term in (6.6) to the first term becomes infinite at the trailing edge of the plate, so that the iterative analysis used to obtain $t_{1}(x)$ is doubtful there. The results obtained in [12] can be applied to Eq. (6.5) and consequently the expression (6.6) gives an uniformly valid solution of Eqs. (6.5). The drag on the plate corresponding to the solution (6.6) is

$$
D=\frac{4 \rho_{0} V_{0}^{2} l}{\sqrt{\pi \sigma}}\left(1+\frac{1.832}{\pi \sqrt{\pi}} \frac{1}{\beta \sqrt{\sigma}}\right) .
$$

ii) The problem of the lift. Due to the singularity $x^{-3 / 2}$ in the first term of the kernel $k_{22}$ the expression (6.4) cannot be substituted directly into Eq. (3.20). But the analysis given in Appendix 1 of [5] can still be used and we obtain the next asymptotic form of the integral 
equation (3.20):

$$
\frac{1}{\pi} \int_{-1}^{1} \frac{t_{2}(\xi)}{x-\xi} d \xi-\frac{1}{2 \sqrt{2 \pi \sigma} \beta} \int_{-1}^{x_{*}} \frac{t_{2}(\xi)}{(x-\xi)^{3 / 2}} d \xi=-\frac{2 \theta}{\beta}
$$

where $\int^{*}$ stands for the Hadamard's principal value of the integral

$$
\int_{-1}^{x_{*}} \frac{f(\xi)}{(x-\xi)^{3 / 2}} d \xi=\lim _{\varepsilon \rightarrow 0}\left\{\int_{-1}^{x-\varepsilon} \frac{f(\xi)}{(x-\xi)^{3 / 2}} d \xi-\frac{2 f(x)}{\sqrt{\varepsilon}}\right\} .
$$

Eq. (6.8) will be solved by successive approximation with respect to the small parameter $\sigma^{-1 / 2}$. By writing $t_{2}(x)=t_{20}(x)+\sigma^{-1 / 2} t_{21}(x)+\cdots$ we obtain the equations

$$
\begin{gathered}
\frac{1}{\pi} \int_{-1}^{1} \frac{t_{20}(\xi)}{x-\xi} d \xi=-\frac{2 \theta}{\beta} \\
\frac{1}{\pi} \int_{-1}^{1} \frac{t_{21}(\xi)}{x-\xi} d \xi=\frac{1}{2 \sqrt{2 \pi} \beta} \int_{-1}^{x_{*}} \frac{t_{20}(\xi)}{(x-\xi)^{3 / 2}} d \xi .
\end{gathered}
$$

The solution of Eq. (6.9) contains an undetermined constant $c$ :

$$
t_{20}(x)=-\frac{2 \theta}{\beta}\left\{\frac{1+c}{2} \sqrt{\frac{1-x}{1+x}}-\frac{1-c}{2} \sqrt{\frac{1+x}{1-x}}\right\} .
$$

Eq. (6.10) becomes

$$
\begin{aligned}
\frac{1}{\pi} \int_{-1}^{1} \frac{t_{21}(\xi)}{x-\xi} d \xi= & -\frac{\theta}{\sqrt{2 \pi} \beta^{2}}\left\{\frac{1+c}{2} \int_{-1}^{x_{*}} \sqrt{\frac{1-\xi}{1+\xi}} \frac{d \xi}{(x-\xi)^{3 / 2}}\right. \\
& \left.-\frac{1-c}{2} \int_{-1}^{x_{*}} \sqrt{\frac{1+\xi}{1-\xi}} \frac{d \xi}{(x-\xi)^{3 / 2}}\right\}
\end{aligned}
$$

For the terms in the right-hand side of this equation we can write the asymptotic forms

$$
\begin{aligned}
& \int_{-1}^{x_{*}} \sqrt{\frac{1-\xi}{1+\xi}} \frac{d \xi}{(x-\xi)^{3 / 2}}=\left\{\begin{array}{l}
\frac{\sqrt{ } 2}{4 \pi}+O(1+x), \\
-\frac{\sqrt{ } 2}{2} \ln (1-x)+O\left((1-x)^{\circ}\right),
\end{array}\right. \\
& \int_{-1}^{x_{*}} \sqrt{\frac{1+\xi}{1-\xi}} \frac{d \xi}{(x-\xi)^{3 / 2}}=\left\{\begin{array}{l}
-\frac{\pi \sqrt{ } 2}{2}+O(1+x), \\
\frac{2 \sqrt{ } 2}{x-1}+\frac{\sqrt{ } 2}{4} \ln (1-x)+O\left((1-x)^{\circ}\right) .
\end{array}\right.
\end{aligned}
$$

In order that $t_{21}$ be integrable on the interval $(-1,1)$ we must have in the right-hand side of Eq. (6.12) at most integrable singularities, so that we must put $c=1$. We obtain

$$
t_{20}(x)=-\frac{2 \theta}{\beta} \sqrt{\frac{1-x}{1+x}},
$$


which coincides exactly with the lift distribution in the classical thin-profile theory by using Kutta-Joukowski postulate. The above analysis is a slight modification of that given by Kusukawa [5] for the case of incompressible fluids.

Now we can write

$$
t_{21}(x)=\frac{1}{\pi} \sqrt{\frac{1-x}{1+x}} \int_{-1}^{1} \sqrt{\frac{1+\xi}{1-\xi}} \frac{g(\xi)}{\xi-x} d \xi
$$

where

$$
g(x)=-\frac{\theta}{\sqrt{2 \pi} \beta^{2}} \int_{-1}^{x_{*}} \sqrt{\frac{1-\xi}{1+\xi}} \frac{d \xi}{(x-\xi)^{3 / 2}} .
$$

In the neighborhood of the trailing edge of the plate we have

and hence

$$
t_{21}(x)=-\frac{\sqrt{2 \pi} \theta}{2 \beta^{2}} \frac{1}{\sqrt{1+x}}+O\left(\sqrt{\frac{1-x}{1+x}}\right)
$$

$$
t_{21}(x): t_{20}(x)=O\left(\frac{1}{\sqrt{\sigma(1-x)}}\right)
$$

Since this ratio becomes infinite at the trailing edge, the above iterative analysis is doubtful there. In the Appendix we focus our attention on the trailing-edge region by using the Wiener-Hopf technique. The results obtained confirm the uniform validity of $t_{2}(x)=t_{20}(x)$ $+\sigma^{-1 / 2} t_{21}(x)$ as an asymptotic solution of the equation (6.8) over the whole plate, and consequently this analysis gives a mathematical justification of the Kutta-Joukowski condition.

The lift on the plate is now

$$
\begin{aligned}
L=2 \pi \rho_{0} V_{0}^{2} l \frac{\theta}{\beta}\left\{1+\frac{1}{(2 \pi)^{3 / 2} \beta \sqrt{ } \sigma} \int_{-1}^{1} \int_{-1}^{x} \sqrt{\frac{1+x}{1-x}} \frac{d \xi d x}{\sqrt{(1+\xi)(x-\xi)}(\sqrt{1-\xi}-\sqrt{1-x})}\right\} \\
\cong 2 \pi \rho_{0} V_{0}^{2} l \frac{\theta}{\beta}\left\{1+\frac{7.72}{(2 \pi)^{3 / 2} \beta \sqrt{ } \sigma}\right\} .
\end{aligned}
$$

The last term in the bracket is a viscosity correction on the lift coefficient in compressible subsonic flow.

By comparing our results for $\beta=1$ with those given by Miyagi [7], the coincidence of the first terms for the drag can be remarked. But the lift coefficient for the plate given by Miyagi for $\sigma \rightarrow \infty$ is half of the lift coefficient for the plate in conventional thin-profile theory to which our formula reduces (6.17) in the limit case of an inviscid fluid.

7. Asymptotic solution for high Reynolds numbers in supersonic flow. In order to derive the asymptotic form of the functions $k_{11}(x)$ and $k_{22}(x)$ for high values of $\sigma$ we use the relation (6.2) and the two following formulae given in $[2,7]$ :

$$
\begin{gathered}
\frac{\sin \varphi}{\pi} \alpha K_{0}(\alpha|x|) e^{\alpha x \cos \varphi}=\delta(x)+O\left(\frac{1}{\alpha}\right), \\
\frac{\operatorname{tg} \varphi}{\pi} K_{1}(\alpha|x|) \operatorname{sgn} x e^{\alpha x \cos \varphi}=\delta(x)+O\left(\frac{1}{\alpha}\right) .
\end{gathered}
$$


The two kernels become

$$
\begin{aligned}
& k_{11}(x)=\frac{\sqrt{ } \sigma}{\sqrt{2 \pi x}} H(x)+\frac{1}{2 \beta_{1}} \delta(x)+O\left(\frac{1}{\sqrt{ } \sigma}\right), \\
& k_{22}(x)=-\frac{H(x)}{4 x \sqrt{2 \pi \sigma x}}+\frac{\beta_{1}}{2} \delta(x)+O\left(\frac{1}{\sigma}\right),
\end{aligned}
$$

where $\beta_{1}=\left(M_{0}^{2}-1\right)^{1 / 2}$.

i) The problem of the drag. By using the form (7.3) of the kernel Eq. (3.19) becomes

$$
\sqrt{\frac{\sigma}{2 \pi}} \int_{-1}^{x} \frac{t_{1}(\xi)}{\sqrt{x-\xi}} d \xi+\frac{1}{2 \beta_{1}} t_{1}(x)=-1, \quad-1<x<1,
$$

and by the change of variables $\xi+1=\xi^{\prime}, x+1=x^{\prime}$,

$$
\sqrt{\frac{\sigma}{2 \pi}} \int_{0}^{x^{\prime}} \frac{t_{1}\left(\xi^{\prime}\right)}{\sqrt{x^{\prime}-\xi^{\prime}}} d \xi^{\prime}+\frac{1}{2 \beta_{1}} t_{1}\left(x^{\prime}\right)=-1 \text { for } 0<x^{\prime}<2 .
$$

Let $T_{1}(s)$ be the LaPlace transform of the function $t_{1}\left(x^{\prime}\right)$. By taking the LaPlace transform of the relation (7.6) we obtain

$$
T_{1}(s)=-\frac{2 \beta_{1}}{\sqrt{ } s\left(\sqrt{ } s+\beta_{1} \sqrt{2 \sigma}\right)} .
$$

The inverse LaPlace transform gives

$$
t_{1}\left(x^{\prime}\right)=-2 \beta_{1} e^{2 \sigma \beta_{1}^{2} x^{\prime}} \operatorname{Erfc}\left(\sqrt{2 \sigma x^{\prime}} \beta_{1}\right)
$$

which expresses the shear force on the plate in the modified variable $x^{\prime}$. By using this expression for $t_{1}\left(x^{\prime}\right)$ we obtain the following formula for the drag:

$$
D=\frac{4 \rho_{0} V_{0}^{2} l}{\sqrt{\pi \sigma}}\left\{1-\frac{\sqrt{ } \pi}{4 \sqrt{\sigma} \beta_{1}}+\frac{\sqrt{ } \pi}{4 \beta_{1} \sqrt{ } \sigma} e^{4 \sigma \beta_{1}^{2}} \operatorname{Erf}\left(2 \beta_{1} \sqrt{ } \sigma\right)\right\} .
$$

ii) The problem of the lift. For large values of the Reynolds number Eq. (3.20) becomes

$$
\beta_{1} t_{2}(x)-\frac{1}{2 \sqrt{2 \pi \sigma}} \int_{-1}^{x_{*}} \frac{t_{2}(\xi)}{(x-\xi)^{3 / 2}} d \xi=-2 \theta, \quad-1<x<1 .
$$

By the same change of variables as above we can write

$$
\beta_{1} t_{2}\left(x^{\prime}\right)-\frac{1}{2 \sqrt{2 \pi \sigma}} \int_{0}^{x_{0}} \frac{t_{2}\left(\xi^{\prime}\right)}{\left(x^{\prime}-\xi^{\prime}\right)^{3 / 2}} d \xi^{\prime}=-2 \theta, \quad 0<x^{\prime}<2 .
$$

This integral equation can be also solved by means of the LaPlace transform. Let $T_{2}(s)=\mathscr{L}\left[t_{2}\left(x^{\prime}\right)\right]$. From $(7.11)$ we obtain

and hence by reversing

$$
T_{2}(s)=\frac{-2 \sqrt{2 \sigma} \theta}{s\left(\sqrt{ } s+\sqrt{2 \sigma} \beta_{1}\right)}
$$

$$
t_{2}\left(x^{\prime}\right)=-\frac{2 \theta}{\beta_{1}}\left\{1-e^{2 \sigma \beta_{1}^{2} x^{\prime}} \operatorname{Erfc}\left(\sqrt{2 \sigma x^{\prime}} \beta_{1}\right)\right\} .
$$


By using this relation we obtain the lift on the plate

$$
L=\frac{4 l \theta \rho_{0} V_{0}^{2}}{\beta_{1}}\left\{1-\frac{1}{\sqrt{\pi \sigma} \beta_{1}}+\frac{1}{4 \sigma \beta_{1}^{2}}+\frac{1}{4 \sigma \beta_{1}^{2}} e^{4 \sigma \beta_{1}^{2}} \operatorname{Erfc}\left(2 \beta_{1} \sqrt{ } \sigma\right)\right\} .
$$

In the limit case $\sigma \rightarrow \infty$ we have

$$
L=\frac{4 l \theta \rho_{0} V_{0}^{2}}{\beta_{1}}
$$

which is the very expression of the lift on the plate in supersonic inviscid fluid flow. The formula (7.14) then gives the viscosity correction on the lift in supersonic flow.

Appendix. We shall investigate now the form of the solution $t_{2}(x)$ of Eq. (6.8) in the trailing edge region. To this end we shift the origin to the trailing edge and perform a stretching of coordinates by means of the transformation

$$
\sigma(\xi-1)=\xi^{\prime}, \quad \sigma(x-1)=x^{\prime} .
$$

Eq. (6.8) becomes:

$$
\frac{1}{\pi} \int_{-2 \sigma}^{0} \frac{t_{2}\left(\xi^{\prime}\right)}{x^{\prime}-\xi^{\prime}} d \xi^{\prime}-\frac{1}{2 \sqrt{2 \pi} \beta} \int_{-2 \sigma}^{x^{\prime}} \frac{t_{2}\left(\xi^{\prime}\right)}{\left(x^{\prime}-\xi^{\prime}\right)^{3 / 2}} d \xi^{\prime}=-\frac{2 \theta}{\beta}, \quad-2 \sigma<x^{\prime}<0 .
$$

For $\sigma \rightarrow \infty$ we take the lower limit of the integrals equal to $-\infty$; likewise, in order to obtain a nontrivial solution we shall put $-(2 \theta / \beta) e^{\delta^{\prime} x^{\prime}}$ for $-2 \theta / \beta$ in the right-hand side of Eq. (A.2). $\delta^{\prime}$ is a small parameter to be determined as a result of the matching process. Eq. (A.2) becomes

$$
\frac{1}{\pi} \int_{-\infty}^{\infty} \frac{\hat{t}_{2}\left(\xi^{\prime}\right)}{x^{\prime}-\xi^{\prime}} d \xi^{\prime}-\frac{1}{2 \sqrt{2 \pi} \beta} \int_{-\infty}^{\infty} \frac{H\left(x^{\prime}-\xi^{\prime}\right) \hat{t}_{2}\left(\xi^{\prime}\right)}{\left(x^{\prime}-\xi^{\prime}\right)^{3 / 2}} d \xi^{\prime}=h\left(x^{\prime}\right)+\hat{g}\left(x^{\prime}\right)
$$

where

$$
\hat{t}_{2}\left(x^{\prime}\right)=t_{2}\left(x^{\prime}\right) H\left(-x^{\prime}\right), \quad h\left(x^{\prime}\right)=-\frac{2 \theta}{\beta} e^{\delta^{\prime} x^{\prime}} H\left(-x^{\prime}\right), \quad \hat{g}\left(x^{\prime}\right)=g\left(x^{\prime}\right) H\left(x^{\prime}\right),
$$

$g\left(x^{\prime}\right)$ being an unknown function.

By taking the Fourier transform of Eq. (A.3) and denoting by capital letters the Fourier transforms we obtain

$$
\lim _{\varepsilon \rightarrow 0}\left\{T_{2}^{+}(p)\left(-i \frac{\sqrt{p+i \varepsilon}}{\sqrt{p-i \varepsilon}}+\frac{1}{\sqrt{2} \beta} \frac{p e^{i(\pi / 4)}}{\sqrt{p-i \varepsilon}}\right)\right\}=-\frac{2 \theta}{\beta} \frac{i}{p+i \delta^{\prime}}+G^{-}(p) .
$$

The superscripts,+- indicate the regularity of the corresponding function in the upper or lower half-plane of the p-plane respectively. We rewrite Eq. (A.4) in the form

$$
\begin{aligned}
& \lim _{\varepsilon \rightarrow 0}\left\{T_{2}^{+}(p)(-i \sqrt{p+i \varepsilon}+\right.\left.\left.\frac{1}{\sqrt{2} \beta} p e^{i(\pi / 4)}\right)+\frac{2 \theta}{\beta} \frac{e^{i(\pi / 4)} \sqrt{\delta^{\prime}+\varepsilon}}{p+i \delta^{\prime}}\right\} \\
&=\lim _{\varepsilon \rightarrow 0}\left\{-\frac{2 \theta}{\beta} i \frac{\sqrt{p-i \varepsilon}-e^{-i(\pi / 4)} \sqrt{\delta^{\prime}+\varepsilon}}{\sqrt{p+i \delta^{\prime}}}+G^{-}(p) \sqrt{p-i \varepsilon}\right\} .
\end{aligned}
$$


Here the left-hand side is a function regular in the upper half $p$-plane and the right-hand side a function regular in the half-plane $\operatorname{Re}\{p\}<0$. Consequently, the ordered couple of these two functions represent a constant on the whole complex $p$-plane. Thus we obtain the function $T_{2}^{+}(p)$ in the form

$$
T_{2}^{+}(p)=\lim _{\varepsilon \rightarrow 0}\left\{-2 \sqrt{2 \theta} \sqrt{\delta^{\prime}+\varepsilon} \frac{\left(p+i \sqrt{2} \beta e^{i(\pi / 4)} \sqrt{p+i \varepsilon}\right)\left(\frac{1}{p+i \delta^{\prime}}+\tilde{c}\right)}{p^{2}-2 \beta^{2} i p+2 \beta^{2} \varepsilon}\right\} .
$$

We choose the constant $\tilde{c}$ so that the point $p=\beta^{2} i+\sqrt{\beta^{4} i-2 \beta^{2} \varepsilon}$ is an eliminable singularity of the function $T_{2}^{+}(p)$. We obtain

$$
T_{2}^{+}(p)=-\frac{4 \beta \theta \sqrt{ } \delta^{\prime}}{2 \beta^{2}+\delta^{\prime}}\left\{\frac{1}{\sqrt{ } 2 \beta} \frac{i}{p+i \delta^{\prime}}-\frac{e^{-i(\pi / 4)}}{\sqrt{ } p\left(p+i \delta^{\prime}\right)}\right\} .
$$

By taking the inverse Fourier transform we get

$$
t_{2}\left(x^{\prime}\right)=-\frac{4 \beta \theta \sqrt{ } \delta^{\prime}}{2 \beta^{2}+\delta^{\prime}}\left\{\frac{1}{\sqrt{ } 2 \beta} e^{\delta^{\prime} x^{\prime}}+\frac{e^{\delta^{\prime} x^{\prime}}}{i \sqrt{ } \delta^{\prime}} \operatorname{Erf}\left(i \sqrt{-\delta^{\prime} x^{\prime}}\right)\right\} .
$$

Now, for small values of $\delta^{\prime}$, in the former variables we obtain

$$
t_{2}(x)=-\frac{2 \theta}{\beta} \sqrt{ } \delta^{\prime}\left\{\frac{2 \sqrt{\sigma(1-x)}}{\sqrt{\pi}}+\frac{1}{\sqrt{ } 2 \beta}+O\left(\delta^{\prime}\right)\right\} .
$$

This solution has to be matched to the "outer" solution obtained in Sec. 6:

$$
t_{2}(x)=-\frac{\sqrt{ } 2 \theta}{\beta} \sqrt{1-x}-\frac{\sqrt{ } \pi \theta}{2 \beta^{2} \sqrt{ } \sigma}+O(1-x) .
$$

Comparison of (A.9) with (A.10) determines the constant $\delta^{\prime}$ to be

$$
\delta^{\prime}=\pi / 8 \sigma .
$$

Moreover, it can be seen that the inner solution (A.9) with $\delta^{\prime}$ given by relation (A.11) agrees with the asymptotic expansion around the trailing edge of the solution given in Sec. 6 . The solution $t_{2}(x)=t_{20}(x)+\sigma^{-1 / 2} t_{21}(x)$ proves to be valid over the whole plate including the trailing edge.

\section{REFERENCES}

[1] L. Bairstow, B. M. Cave and E. D. Lang, Phil. Trans. Roy. Soc. (London) A 223, 383 (1923)

[2] L. Dragoş and N. Marcov, J. Mécanique 7, 379 (1968)

[3] D. Homentcovschi, Arch. Mech 27, 325. (1975)

[4] D. Homentcovschi, Bull. Math. Soc. Sci. Math. R.S.R. 19, 57(1976)

[5] K. Kusukawa, J. Phys. Soc. Japan 19, 1031 (1964)

[6] J. A. Levis and G. F. Carrier, Quart. Appl. Math. 7, 228 (1949)

[7] T. Miyagi, J. Phys. Soc. Japan 19, 1063 (1964)

[8] N. A. V. Piercy and H. F. Winny, Proc. Roy. Soc. A 140, 543 (1933)

[9] R. Seebass, K. Tamada and T. Miyagi, Phys. Fluids 9, 1697 (1966)

[10] L. Sirovich, Phys. Fluids 11, 1424 (1968)

[11] K. Stewartson, Proc. Roy. Soc. A 277, 107 (1963)

[12] K. Tamada and T. Miyagi, J. Phys. Soc. Japan 20, 454 (1965) 